\title{
Computer Axisymmetric Model of a Piezoelectric Micropump
}

\author{
Ildar Sh. Nasibullayev ${ }^{1, *}$, Elvira Sh. Nasibullaeva ${ }^{1}$ and Oleg V. Darintsev ${ }^{1,2}$ \\ ${ }^{1}$ Mavlyutov Institute of Mechanics UFRC RAS, Ufa 450054, Russian Federation \\ ${ }^{2}$ Ufa State Aviation Technical University, Ufa 450008, Russian Federation
}

Received 17 November 2020; Accepted 10 April 2021

\begin{abstract}
A computer axisymmetric model, in which periodic vibrations of ring piezoelectric actuators placed on an elastic tube of circular cross-section lead to radial deformations of the tube, has been constructed in the form of the FreeFem++ solver. As a result of these deformations, a local compression of the microchannel occurs with a change in its volume and the corresponding expulsion of the fluid, which induces a flow inside it. The purpose of the work is to study the influence of oscillations of piezoelectric actuators on the fluid flow in a microchannel in respect to the development of a new technical device, such as a piezoelectric micropump, as well as to carry out a numerical analysis of its operation modes. The advantages of the proposed micropump are simplicity of design with the possibility of expanding its functionality; small size; fluid flow rate sufficient to supply coolant to the micro-gripper chamber; a flexible system for controlling the average fluid flow rate using the number of piezoelectric elements involved and their vibration frequency. When testing a computer model, the main sources of errors and the limits of applicability of the model were identified. Using the developed profiler, the distribution of CPU time is determined depending on the type of physical and optimization problems being solved. To reduce the calculation errors, an irregular (matched with the velocity profile) and adaptive (reconstructed at each time step) computational mesh for the microchannel was constructed so that the boundary elements on the inner side of the tube coincided with the boundary elements of the inner surface of the microchannel. Numerical analysis showed that when using an asymmetric scheme for oscillation of a piezoelectric actuators system, it is possible to achieve a non-zero average fluid flow rate. The influence of the Dirichlet and Neumann boundary conditions on the generated flow is considered, and the propagation of heat into micropipe during its contact with the thermostat is analyzed. It was found that the average fluid flow rate is proportional to the number of piezoelectric actuators and their frequency; the Dirichlet boundary conditions in comparison with the Neumann ones give a significant increase in the average fluid flow rate. An analytical formula that connects the average liquid flow rate with the parameters of the micropump operation mode (the number of piezoelectric actuators and their frequency) is derived. This formula can be used to calculate the parameters of the operating mode in the software module of the device control system in real time.
\end{abstract}

Keywords: axisymmetrical multi-physics computer simulation, fluid-structure interaction, ring piezoelectric actuator, micropump, microgripper cooling system, FreeFem++

\section{Introduction}

Recently, mathematical and computer modeling of microfluidic systems has been in demand and is actively advancing in connection with the development of new types of microdevices [1]. The creation of new technical devices becomes possible due to the peculiarities of fluid flow in microsystems: as the scale of the system decreases, the Reynolds Re and Péclet Pe numbers that characterized the dynamic properties of the system become small. A small Reynolds number $(\operatorname{Re}<10)$ implies a laminar flow, corresponding to both most technical microdevices and biological systems [2]. Consequently, it becomes possible to develop efficient systems for supplying and dosing fluid [3], as well as miniature cooling systems [4]. A small Péclet number means that during heat transfer the contribution of molecular thermal conductivity prevails over convective heat transfer, therefore, in microdevices (microheat

*E-mail address: sp.ishn@gmail.com

ISSN: 1791-2377@ 2021 School of Science, IHU. All rights reserved. doi:10.25103/jestr.142.19 exchanger, cooling system, micro-gripper) in contrast to macrosystems both mechanisms of heat transfer must be taken into account [5]. The basic performance characteristics of microdevices largely depend on the principle of operation and design of micropumps as well as hydraulic resistances. So, ultra-small cross-sections of channels require a more careful adjustment of the boundary conditions between the liquid and the working elements in the complete absence of the pollution factor. Therefore, the most widespread are peristaltic pumps since in them only the deformable element has contact with the working fluid [6]. However, when calculating operating modes, only empirical formulas are used that do not take into account the specifics of the application and the scale factor, the magnitude of the pulsations is not analyzed, which requires the development of accurate theoretical models.

The intensive growth in the production of microelectromechanical systems [7] and the widespread introduction of microrobots [8] are a significant incentive for the development of new advansed devices that ensure careful handling of microcomponents [9]. The authors of this 
work have developed and are investigating a capillary micro-gripper intended for manipulating flat micro-objects and membranes with a sufficient area of a flat side, which cannot be held by finger-gripper or other types of grippers [10]. The creation of a working model of this micro-gripper, built on the basis of a miniature Peltier element, requires a cooling system to remove heat from the hot side of the Peltier element. Studies by Afshari [11] showed that with a decrease in the size of the heat-exchange chamber, the efficiency of a fluid cooling system in comparison with an air one increases. Removal of excess heat power from the Peltier element requires the development of a micropump design. Ensuring the stability of the micro-gripper operation and the correct synthesis of control rules require the development of an appropriate mathematical and computer model.

The synthesis of the control system [12] of the capillary micro-gripper is a complex problem. It is requiring, first of all, the solution of the problem of obtaining reliable and complete information about the state of the environment and the capture itself. The miniature design of the device does not allow the use of most sensors typical for robots in the classic scale design. The only measuring instruments that could be integrated into the micro-gripper are temperature sensors, with the help of which the operator or the control system obtains information on the degree of heating of the "hot" side of the Peltier element and the cooling of the working surface [13]. Ambient temperature and humidity information is also available through the integration of digital temperature and humidity sensors into the work environment. This information is not enough to improve the performance of the micro-gripper, therefore there is a high demand for accurate models describing the operation of the device and auxiliary equipment. Condensation of moisture from the air occurs only on surfaces cooled below the dew point, while the cooling rate and the accuracy of maintaining the required temperature directly depend on the efficiency of the cooling system [13]. In order to reduce the time required for the formation of a liquid film during capture and subsequently remove it when releasing a micro-object, it is necessary to implement such a micro-gripper operation mode when the temperature of its working surface is in a vicinity of the operating point (an indirect estimate indicates the required range of $0.5-1^{\circ} \mathrm{C}$ ), namely the dew point. To synthesize such a high-precision pump performance control system, which ensures the stability of the Peltier element, its precise characteristics are required. Therefore relevant is the realization of complex, computational and natural experiments, a good basis for which will serve as an adequate computational model implemented without the use of empirical data and take full account of the specificity of microfluidic systems. Since the final version of the control system is planned to be implemented as an intelligent system $[14,15]$, then for its training, adjustment and verification due to the absence of measured parameters, it will be necessary to use the calculated model data.

Note also that the known examples of pumps are designed taking into account the specifics of the field of application [16] and are fairly large-scale devices. From the existing line of pumps it is impossible to select a micro pump for the micro-gripper cooling system, which has a small size, sufficient fluid flow and a flexible fluid flow control system. Besides, the theoretical models developed for these micropumps are based on empirical data, which does not allow a performed geometric design optimization and determine the optimal operating modes. On the other hand, the generation of a fluid flow in an elastic microchannel belongs to the field of multiphysics, namely, fluid-structure interaction (FSI) [17], which requires, when developing new technical devices, the construction of new mathematical models connecting hydrodynamics, elasticity theory, heat transfer process, parametric optimization and device control theory [18].

With the development of mathematical and computer models, the finite element method (FEM) [19] numerical simulation software have appeared, which allow modeling devices with almost arbitrary geometry [20]. Chiang et al. [21] developed an axisymmetric FSI computer model for the interaction of a Newtonian fluid with a hyperelastic incompressible body and analyzed the stability of differential equations written in variational form using FEM modeling within the framework of the FreeFem++ numerical simulation software [22].

The authors of present work propose the idea of a piezoelectric micropump consisting of an elastic tube with system of the ring piezoelectric actuators (PEAs) [23]. Known models of fluid flow through static hydraulic resistance [20] are difficult to adapt to simulate this piezoelectric micropump. Therefore, algorithms for modeling fluid flow in a channel with a dynamically variable geometry have been developed [24]. In the work [25], the deformation of the tube was calculated using the equations of elasticity, which were solved on its wall. In the subsequent work [26], a mathematical and computer model of a fluid flow regulator is presented using the dynamic forming of hydraulic resistance by compressing an elastic tube with a PEA. A model of a micropump that creates a fluid flow (with a flow rate up to $50 \mathrm{mkl} / \mathrm{s}$ ) in a flat channel using immersed in it bending PEA was developed [27]. Finally, in the work [28], an axisymmetric computer model of a piezoelectric micropump was proposed. It is shown that an asymmetric oscillation scheme of a system of PEAs creates a nonzero average fluid flow in a tube even in the absence of an external pressure gradient applied along the channel. This model can be used in the development of a micropump operating in a closed loop, and become the basis for the synthesis method of the software part of its control system for the fluid cooling system of the micro-gripper.

To determine the parameters of the operating mode of a technical device (or change the parameters when switching from one mode to another), it is necessary to carry out a complete numerical simulation of the device using a multiparameter set of input data. By the full factorial computational experiment method [29], it is possible to determine the degree of influence of the input parameters and their mutual influence on the output parameters of the device operating mode. The use of factorial computational experiments of the second order reveals a nonlinear relationship between the parameters [30]. The result of a factorial computational experiment is an analytical function corresponding with certain accuracy to a complete numerical simulation, which can be used in the software of the device control system in real time [31].

This paper proposes an extended in comparison with work [28] axisymmetric computer model of a piezoelectric micropump consisting of an elastic tube and a system of ring PEAs with an analysis of its operation modes. This model takes into account the boundary conditions of both in Dirichlet and Neumann form as well as the processes of fluid heat transfer. The advantages of the proposed micropump model are simplicity of design with the possibility of expanding its functionality (change in the 
number of PEAs, installation of microvalves, etc.); small dimensions (the length of the micropump is $10 \mathrm{~mm}$, and the radius is $5 \mathrm{~mm}$ ); fluid flow rate sufficient to cool the microgripper; a flexible control system of the average liquid flow rate by the number of PEAs involved and their vibration frequency.

\section{Problem formulation and governing equations}

An axisymmetric flow of a fluid through an elastic tube with internal $R_{1}$ and external $R_{2}$ radii and length $L$ is investigated. A system of annular PEAs of length $\ell$ is located coaxially on the tube. In Fig. 1( $a$ ) shows the geometry of the problem and introduced the following notation: $\Gamma_{1}$ and $\Gamma_{2}$ are channel inlet and outlet, respectively; $\Gamma_{3}$ is axis of symmetry; $\Gamma_{4}, \Gamma_{5}$ и $\Gamma_{6}$ are inner, side and outer walls of the tube, respectively; $\Gamma_{i}^{p}$ is contact boundary of the $i$-th PEA with the outer wall of the tube. The dotted lines indicate the geometry extension for the heat problem: $\Gamma_{7}$ is the thermostat (TS) contact boundary with the tube; $\Gamma_{0}, \Gamma_{8}$ are channel inlet and outlet, respectively. The cylindrical coordinate system contains the radial $\mathrm{Or}$ and axial $\mathrm{Oz}$ axes of the tube with the origin located at its geometric center. The initial prototype of the micropump is shown on the Fig. $1(b)$. The CAD model of the updated micropump shell is shown on Fig. 1(c) (up part) and Fig. 1 $(d)$ (down part).

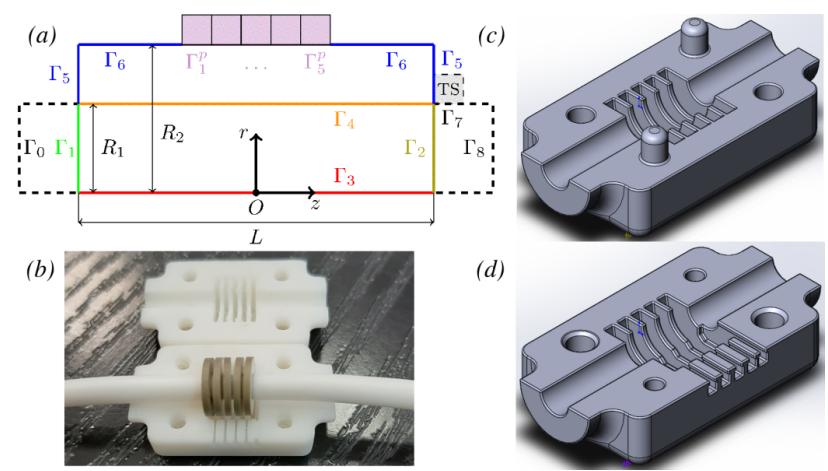

Fig. 1. Geometry and basic notations of a model $(a)$; prototype of the micropump $(b)$; CAD model of the up $(c)$ and down $(d)$ parts of the updated micropump shell.

An axisymmetric fluid flow is described by the NavierStokes and incompressibility equations [32]

$\rho\left(\frac{\partial u_{r}}{\partial t}+u_{r} \frac{\partial u_{r}}{\partial r}+u_{z} \frac{\partial u_{r}}{\partial z}\right)=$
$-\frac{\partial p}{\partial r}+\mu\left[\frac{1}{r} \frac{\partial}{\partial r}\left(r \frac{\partial u_{r}}{\partial r}\right)+\frac{\partial^{2} u_{r}}{\partial z^{2}}-\frac{u_{r}}{r^{2}}\right]$,

$\rho\left(\frac{\partial u_{z}}{\partial t}+u_{r} \frac{\partial u_{z}}{\partial r}+u_{z} \frac{\partial u_{z}}{\partial z}\right)=$

$-\frac{\partial p}{\partial z}+\mu\left[\frac{1}{r} \frac{\partial}{\partial r}\left(r \frac{\partial u_{z}}{\partial r}\right)+\frac{\partial^{2} u_{z}}{\partial z^{2}}\right]$,

$\frac{1}{r} \frac{\partial}{\partial r}\left(r u_{r}\right)+\frac{\partial u_{z}}{\partial z}=0$

where $\rho$ is volume density of fluid; $\mathbf{u}=\left(u_{r}, u_{z}\right)$ is fluid velocity consisting of radial and axial components; $t$ is time; $p$ is pressure; $\mu$ is fluid dynamic viscosity.

Channel deformations are described by the Lamé equations [33]

$2(1-v)\left(\frac{\partial^{2} s_{r}}{\partial r^{2}}+\frac{1}{r} \frac{\partial s_{r}}{\partial r}-\frac{s_{r}}{r^{2}}\right)+\frac{\partial^{2} s_{z}}{\partial r \partial z}+$
$(1-2 v) \frac{\partial^{2} s_{r}}{\partial z^{2}}=0$

$2(1-v) \frac{\partial^{2} s_{z}}{\partial z^{2}}+\left(\frac{\partial^{2} s_{r}}{\partial r \partial z}+\frac{1}{r} \frac{\partial s_{r}}{\partial z}\right)+$

$(1-2 v)\left(\frac{\partial^{2} s_{z}}{\partial r^{2}}+\frac{1}{r} \frac{\partial s_{z}}{\partial r}\right)=0$,

where $\mathbf{s}=\left(s_{r}, s_{z}\right)$ is the deformation vector consisting of radial and axial components; $\lambda$ and $\eta$ are the Lamé coefficients related to Young's modulus of elasticity $E$ and Poisson's ratio $v$ by the expressions

$$
\lambda=\frac{v E}{(1+v)(1-2 v)}, \quad \eta=\frac{E}{2(1+v)} .
$$

Note that the stationary Lamé equations (4) and (5) are applicable for the oscillation frequency of PEAs up to $f_{e}=100 \mathrm{kHz}$ since the elastic relaxation time is $\tau_{e}=R_{1} \sqrt{\rho_{e} / E} \approx 10^{-7} \mathrm{~s}$, where $\rho_{e}$ is the tube material density. For a higher frequency, it is necessary to consider the dynamic equations. In real PEA the highest operating frequency is limited by the resonance frequency $f_{r}$ which is usually lower than $f_{e}$ (lead zirconate titanate (PZT) ring PEAs has $f_{r}$ order of $20 \ldots 30 \mathrm{kHz}[34], 25 \mathrm{kHz}$ [35], $50 \mathrm{kHz}$ [36], 40...90 kHz [23]). Thus the upper limit for the frequency, when the model applicable, is defined as $\min \left(f_{e}, f_{r}\right)$. The relative deformations are up to $0.28 \%$ so the error of linear equations is small.

Since the investigated design of the micro-pump is supposed to be used in the micro-gripper cooling system, then to check the degree of influence of the heated liquid from the micro-gripper on the cooling fluid of the microchannel, the equation of thermal conductivity is solved [32]:

$c_{p} \rho\left(\frac{\partial T}{\partial t}+(\mathbf{u} \cdot \nabla) T\right)=$
$\frac{1}{r} \frac{\partial}{\partial r}\left(\lambda_{T}(T) r \frac{\partial T}{\partial r}\right)+\frac{\partial}{\partial z}\left(\lambda_{T}(T) \frac{\partial T}{\partial z}\right)$,

where $T$ is the fluid temperature; $c_{p}$ is the isobaric specific heat of fluid; $\nabla$ is the nabla operator; $\lambda_{T}$ is the coefficient of thermal conductivity depending on the temperature of the fluid.

Equations (1)-(6) were solved numerically by FEM in the FreeFem++ package [22], where the integration is performed in Cartesian coordinates over the area element $\mathrm{d} S=\mathrm{d} x \mathrm{~d} y$. Therefore, it is necessary to transform a given area element into cylindrical coordinates (with an area element $\mathrm{d} \Omega=\mathrm{d} z \mathrm{~d} r$ ) according to the rule $\mathrm{d} S=J \mathrm{~d} \Omega$, according to the rule $J=r$ is the Jacobian of the transformation of Cartesian coordinates into cylindrical. Time discretization for the heat equation was carried out by the implicit Euler method [37] of the first order $\mathrm{d} x^{n+1} / \mathrm{d} t=\left(x^{n+1}-x^{n}\right) / \tau$, where for the variable $x$ the indices $n+1$ and $n$ refer to the current and previous moment in time; $\tau$ is the time step.

The performed numerical analysis showed that when using the Euler scheme, the axisymmetric variational form of the Navier-Stokes equations quickly becomes unstable. 
Ildar Sh. Nasibullayev, Elvira Sh. Nasibullaeva and Oleg V. Darintsev/

Journal of Engineering Science and Technology Review 14 (2) (2021) 152 - 164

The stability of the scheme is provided by the method of characteristics-Galerkin for the approximation of the total time derivative $[21,22]$

$\frac{\partial \mathbf{u}}{\partial t}+(\mathbf{u} \cdot \nabla) \mathbf{u}=\frac{\mathbf{u}^{n+1}-\mathbf{u}^{n} \circ \mathbf{X}^{\mathbf{n}}}{\tau}$

Multiplying the equations of fluid dynamics (1)-(3) by the Jacobian $r$, an axisymmetric variational form can be obtained

$$
\begin{aligned}
\int\left[\left(\frac{\mathbf{u}^{n+1}-\mathbf{u}^{n} \circ \mathbf{X}^{\mathbf{n}}}{\tau}\right.\right. & \left.+\nabla p^{n+1}\right) \mathbf{w} \\
& +\mu\left(\frac{\partial \mathbf{u}^{n+1}}{\partial r} \frac{\partial \mathbf{w}}{\partial r}+\frac{\partial \mathbf{u}^{n+1}}{\partial z} \frac{\partial \mathbf{w}}{\partial z}+\frac{u_{r}^{n+1}}{r^{2}} w_{r}\right) \\
& +\left(\mathbf{u}^{n+1} \cdot \nabla w_{p}+\frac{u_{r}^{n+1}}{r} w_{p}\right. \\
& \left.\left.+\varepsilon_{p} p^{n+1} w_{p}\right)\right] r \mathrm{~d} \Omega=0
\end{aligned}
$$

where $\mathbf{w}=\left(w_{r}, w_{z}\right)$ and $w_{p}$ are trial functions. The corresponding FreeFem++ code is as follow:

Code 1. Navier-Stokes equations.

$/ /$ [uz,ur,p] - variables

$/ /[\mathrm{vz}, \mathrm{vr}, \mathrm{vp}]$ - trial functions

// ThF - channel mesh

$/ / x$ denotes the axial component of $z$

$/ /$ and $y$ the radial component of $r$

// convect - Characteristics-Galerkin Method

problem NavierStokesAS

([uz,ur,p],[vz,vr,vp],eps=1.e-10, solver $=$ Crout $)=$ int $2 \mathrm{~d}(\mathrm{ThF})($

rho/tau*( uz*vz + ur*vr)*y

$+\operatorname{muf} *(\mathrm{dx}(\mathrm{uz}) * \mathrm{dx}(\mathrm{vz})+\mathrm{dy}(\mathrm{uz}) * \mathrm{dy}(\mathrm{vz})$

$\left.+\mathrm{dx}(\mathrm{ur}) * \mathrm{dx}(\mathrm{vr})+\mathrm{dy}(\mathrm{ur})^{*} \mathrm{dy}(\mathrm{vr})+\mathrm{ur} * \mathrm{vr} / \mathrm{y}^{\wedge} 2\right)^{*} \mathrm{y}$

$-\left(p^{*} d x(v z)+p * d y(v r)\right) * y$

$+(d x(u z) * v p+d y(u r) * v p+u r * v p / y$

$\left.\left.+\mathrm{p}^{*} \mathrm{vp} \mathrm{p}^{*} \mathrm{epsp}\right)^{*} \mathrm{y}\right)$

+ int $2 \mathrm{~d}(\mathrm{ThF})($

-rho/tau*convect([upz,upr],-tau,upz)*vz*y

-rho/tau*convect([upz,upr],-tau,upr)*vr*y)

Similarly, for elasticity equations (4) and (5), using the replacement

$r \frac{\partial^{2} u_{i}}{\partial r^{2}}+\frac{\partial u_{i}}{\partial r}=\frac{\partial}{\partial r}\left(r \frac{\partial u_{i}}{\partial r}\right)$

one can get:

$$
\begin{aligned}
\int[(\lambda+2 \eta)(\nabla \mathbf{s} \cdot & \left.\nabla \mathbf{v}+\frac{s_{r}}{r^{2}} v_{r}\right)+(\lambda \\
& +\eta)\left(\frac{\partial s_{z}}{\partial r} \frac{\partial v_{r}}{\partial z}+\frac{\partial s_{r}}{\partial r} \frac{\partial v_{z}}{\partial z}-\frac{\partial s_{r}}{\partial z} v_{z}\right) \\
& \left.+\eta\left(\frac{\partial s_{r}}{\partial z} \frac{\partial v_{r}}{\partial z}+\frac{\partial s_{z}}{\partial r} \frac{\partial v_{z}}{\partial r}\right)\right] r \mathrm{~d} \Omega=0
\end{aligned}
$$

where $\mathbf{v}=\left(v_{r}, v_{z}\right)$ are trial functions with the corresponding FreeFem++ code:

Code 2. Lame equations. problem LameAS $([\mathrm{sz}, \mathrm{sr}],[\mathrm{vvz}, \mathrm{vvr}], \mathrm{eps}=1 . \mathrm{e}-10$, solver $=\mathrm{LU})=$ int $2 \mathrm{~d}(\mathrm{ThE})($

$(\mathrm{lambda}+2.0 * \mathrm{mu}) *(\mathrm{dy}(\mathrm{sr}) * \mathrm{dy}(\mathrm{vvr}) * \mathrm{y}+\mathrm{sr} / \mathrm{y} * \mathrm{vvr})$

$+($ lambda $+\mathrm{mu}) * \mathrm{dy}(\mathrm{sz}) * \mathrm{dx}(\mathrm{vvr}) * \mathrm{y}$

$+\mathrm{mu}^{*} \mathrm{dx}(\mathrm{sr}) * \mathrm{dx}(\mathrm{vvr}) * \mathrm{y}$

$+($ lambda $+2.0 * \mathrm{mu}) * \mathrm{dx}(\mathrm{sz}) * \mathrm{dx}(\mathrm{vvz}) * \mathrm{y}$

$+(\mathrm{lambda}+\mathrm{mu}) *(\mathrm{dy}(\mathrm{sr}) * \mathrm{dx}(\mathrm{vvz}) * \mathrm{y}$

$\left.-\mathrm{dx}(\mathrm{sr})^{*} \mathrm{vvz}\right)$

$+\mathrm{mu} * \mathrm{dy}(\mathrm{sz}) * \mathrm{dy}(\mathrm{vvz}) * \mathrm{y})$

Axisymmetric variational form of the heat conduction equation (6)

$$
\begin{gathered}
\int\left[c_{p} \rho\left(\frac{T^{n+1}-T^{n}}{\tau}+(\mathbf{u} \cdot \nabla) T^{n+1}\right) v_{T}+\lambda \nabla T^{n+1}\right. \\
\left.\cdot \nabla v_{T}\right] r \mathrm{~d} \Omega=0
\end{gathered}
$$

where $v_{T}$ is a trial function with the corresponding FreeFem++ code:

Code 3. Heat equation.

problem HeatAS

$(\mathrm{Tf}, \mathrm{vT}, \mathrm{eps}=1 . \mathrm{e}-10$, solver $=\mathrm{UMFPACK})=$

int $2 \mathrm{~d}(\mathrm{ThF})(\mathrm{cp} *$ rho * Tf/tau*vT * y

+ cp*rho* $\left(\mathrm{uz}^{*} \mathrm{dx}(\mathrm{Tf}) * \mathrm{vT}+\mathrm{ur} * \mathrm{dy}(\mathrm{Tf}) * \mathrm{vT}\right) * \mathrm{y}$

$+\operatorname{lambdaT} *(\mathrm{dx}(\mathrm{Tf}) * \mathrm{dx}(\mathrm{vT})+\mathrm{dy}(\mathrm{Tf}) * \mathrm{dy}(\mathrm{vT})) * \mathrm{y})$

$+\operatorname{int} 2 \mathrm{~d}(\mathrm{ThF})\left(-\mathrm{cp}^{*}\right.$ rho* Tfp/tau*vT*y)

The initial conditions correspond to the absence of deformations on the outer wall $\mathbf{s}=0$ and the fluid at rest $\mathbf{u}=0$.

The boundary conditions for the fluid velocity are as follows:

$\Gamma_{1}: u_{r}=0, p=-\Delta p / 2$,

$\Gamma_{2}: u_{r}=0, p=\Delta p / 2$,

$\Gamma_{3}: u_{r}=0$,

$\Gamma_{4}: u_{r}=0, u_{z}=0$,

where $\Delta p$ is the pressure difference at the inlet and outlet of the tube.

The microtube is fixed at the ends

$\Gamma_{5}: s_{r}=s_{z}=0$.

Neumann boundary conditions (NBC) for deformations are determined from the equilibrium condition $\sigma_{r r}=$ $-p_{i}^{p}(t)$, where the stress tensor component $\sigma_{r r}$ is balanced by the pressure $-p_{i}^{p}(t)$ exerted on the tube by the $i$-th PEA at the boundary $\Gamma_{i}^{p}$ :

$\int\left[(\lambda+2 \eta) \frac{\partial s_{r}}{\partial r} v_{r}+\lambda \frac{s_{r}}{r}+\lambda \frac{\partial s_{z}}{\partial z}-p_{i}^{p}(t)\right] r d \Omega=0$.

Since the ends of the micropipe are fixed (7), then in condition (8) it is necessary to prohibit the transverse displacements $u_{z}=0$, therefore, the NBC will take the following form

$\int\left[(\lambda+2 \eta) \frac{\partial s_{r}}{\partial r} v_{r}+\lambda \frac{s_{r}}{r}-p_{i}^{p}(t)\right] r d \Omega=0$.

When using the Dirichlet boundary conditions (DBC), the displacements of the PEAs are specified by fixed values $s_{r, i}^{p}(t)$, and the DBC are of the form

$\Gamma_{i}^{p}: s_{r}=s_{r, i}^{p}(t) \quad(i=1, \ldots, N)$.

For the stability of the numerical scheme, an additional 
condition is introduced on the boundary $\Gamma_{3}: \mathbf{s}=0$.

Let's note the physical meaning of NBC and DBC. NBC stands for "soft" PEA, the shape of which changes during compression (but the contact area does not change), and DBC stands for "hard" PEA, the shape of which does not change during compression.

The dependence of the pressure of the PEA on the outer wall of the microchannel is determined as $p^{p}(t)=\max \left(p^{p}\right) f_{p}(t)$, and displacements are determinated in the form $s_{r, i}^{p}(t)=s_{r}^{p} f_{p}(t)$, where signal form $\left.f_{p}(t)\right)$ is a harmonic function normalized in the region $[0,1]$ with an oscillation frequency $f$

$f_{p}(t)=[1-\cos (2 \pi f t)] / 2$.

Boundary conditions for the heat conduction equation are

$\Gamma_{1}: T=T_{0} ; \Gamma_{7}: T=T_{1}$.

where $T_{0}$ is the ambient temperature and $T_{1}$ is the TS temperature.

\section{Numerical simulation}

Numerical simulation was carried out for an elastic tube with a length of $L=10 \mathrm{~mm}$ with a circular cross section, an inner radius of $R_{1}=1 \mathrm{~mm}$ and an outer radius of $R_{2}=1.75 \mathrm{~mm}$. Ring PEAs with $\ell=1 \mathrm{~mm}$ were located symmetrically relative to the center of the tube. Configurations with one, three and five PEAs were considered $\left(N_{p}=1,3,5\right)$. The tube material is silicone rubber with Young's modulus $E=5 \mathrm{MPa}$ and Poisson's ratio $\sigma=0.49$. Water is used as a working fluid at a temperature of $T_{0}=20^{\circ} \mathrm{C}$ with a volume density $\rho=998 \mathrm{~kg} / \mathrm{m}^{2}$ and a dynamic viscosity $\mu=1.002 \mathrm{mPa} \cdot \mathrm{s}$.

\subsection{Verification of computer model}

The fluid flow in the channel can be created in two ways: due to the pressure difference $\Delta p$ applied to the channel and due to the periodic simultaneous oscillations of the $N_{p}$ PEAs. Modeling occurs on two matched 2D meshes on a plane $\mathrm{Ozr}$. First, the equations of elasticity are solved on the mesh defining the tube wall. At each time step according to the obtained deformation field, the mesh for the channel is remeshed since the inner surface of the tube, deformed by PEAs, determines the outer boundary of the channel. Then the equations of hydrodynamics and heat conduction are solved on the rearranged channel mesh.

As a calculation parameter, the radial compression amplitude, identical for all PEAs, was set as $s_{r, i}^{p}=s_{p}$, which was directly used in the DBC (10). In order to provide the same amplitude in NBC (9) [25], a preliminary calculation was carried out with the simultaneous compression of all PEAs with a pressure amplitude $p_{0}$ and the value of compression $s_{0}$ was determined. Since the elasticity equations are linear, the value of the pressure $p_{p}$, at which the PEA will be compressed by $s_{p}$ defined as $p_{p}=p_{0} \cdot s_{p} / s_{0}$. The result is a calculated value of $p_{p}=221 \mathrm{kPa}$.

A feature of the problem under consideration is that the deformations of the microchannel are small $(0.28 \%$ of the radius) and at high vibration frequencies the fluid flow rate is high (several $\mathrm{mm} / \mathrm{s}$ ). Consequently, large errors of numerical calculation are possible, depending on the time step $\tau$ and the size $\ell_{m}$ of the mesh elements. Let's denote by $M\left(m, n, N_{p}\right)$ the simulation parameters, where $m$ is the number of boundary elements of the tube mesh per one PEA; $n$ is the number of time steps for each half-period of oscillations of the PEA; $N_{p}$ is the number of PEAs. The meshes for the microchannel and the tube are consistent: the boundary elements on the contact line of the meshes coincide.

\subsection{Mesh}

First, we investigate the symmetric case with one PEA $N_{p}=1$ for $f=1 \mathrm{kHz}$ on the mesh $M(12,40,1)$. On the Fig. 2 the velocity profiles are shown at times $t=T_{f} / 4$ and $t=3 T_{f} / 4$, when the velocity modulus $\left|u_{z}\right|$ is at its maximum, as well as $t=0$ and $t=T_{f} / 2$, when the direction of compression of the PEA changes. Here $T_{f}=1 / f$ is the oscillation period of the PEA with frequency $f$.

It can be seen that the main change in the axial velocity $u_{z}$ occurs near the tube boundary on the interval $r=\left[0.65 R_{1}, R_{1}\right]$. Therefore, to improve the calculation accuracy, it is necessary to use a non-uniform fine mesh near the inner tube boundary. To reduce the computation time, the mesh near the tube axis, where the velocity profile is almost flat, can be made more rarefied. In the modified mesh $M(n, m, 1)$, the size of elements on the inner surface is $\ell_{m}=L /(10 \cdot n)$, and on the axis is $\ell_{m}=L /(5 \cdot n)$ (see Fig. 3).

When the PEAs are deformed, the channel geometry changes and, as a result, it is necessary to rebuild the channel mesh at each time step. In the process of remeshing, a situation may arise when finite elements, after moving, compress adjacent elements by an amount exceeding their size, which leads to the appearance of elements with a negative volume and an emergency interruption of the program.

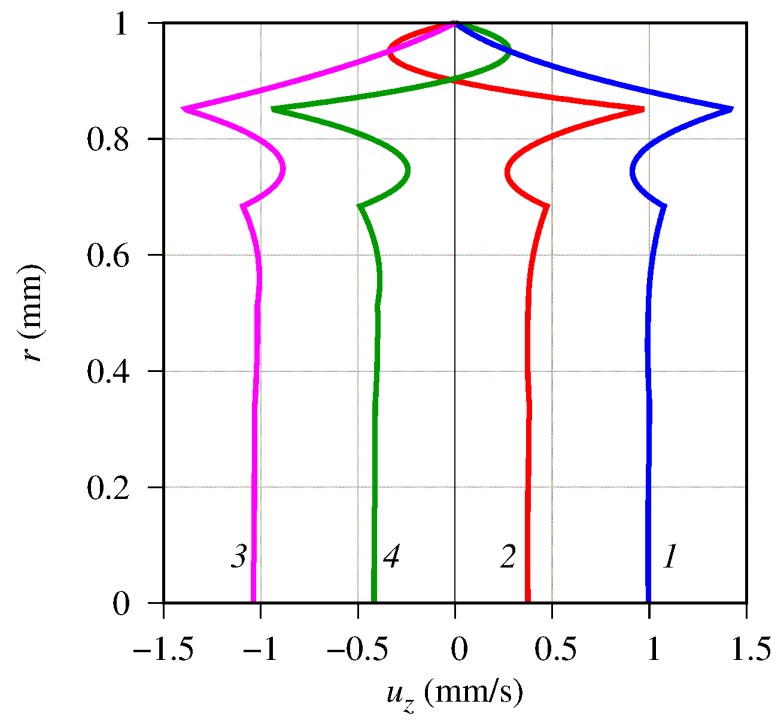

Fig. 2. Outlet velocity profiles $u_{z}$ at the times: $t=0(1), t=T_{f} / 4$ (2), $t=T_{f} / 2(3), t=3 T_{f} / 4$ (4) for parameters: $N_{p}=1 ; f=1 \mathrm{kHz}$.

To prevent this situation, the following algorithm is proposed: full displacement of finite element nodes is replaced by partial displacements by the value of the deformation vector $\left[s_{z}, s_{r}\right]$ in the contact area of the channel mesh and the tube mesh $r=r I n$ with a coefficient $0<c \leq 1$ preventing the appearance of negative volumes. The displacement coefficient is determined automatically by halving until the move is "safe". After each partial displacement, the mesh was adapted. Partial displacements continue until they add up to a full displacement. Algorithm code in FreeFem++ language (algorithm for Cartesian coordinates is presented in the paper [27]) as follows: 
Ildar Sh. Nasibullayev, Elvira Sh. Nasibullaeva and Oleg V. Darintsev/

Journal of Engineering Science and Technology Review 14 (2) (2021) 152 - 164

Code 4. Safe remeshing.

real $\mathrm{t}=1.0$;

real $\mathrm{c}=1.0$

while $(\mathrm{t}>0)\{$

if (checkmovemesh $(\mathrm{ThF},[\mathrm{x}+\mathrm{c} * \mathrm{sz}(\mathrm{x}, \mathrm{rIn})$,

$\mathrm{y}+\mathrm{c} * \operatorname{sr}(\mathrm{x}, \mathrm{rIn}) * \mathrm{y} / \mathrm{rIn}])>0.0)\{$

$\mathrm{ThF}=\operatorname{movemesh}(\mathrm{ThF},[\mathrm{x}+\mathrm{c} * \mathrm{sz}(\mathrm{x}, \mathrm{rIn})$,

$\mathrm{y}+\mathrm{c} * \operatorname{sr}(\mathrm{x}, \mathrm{rIn}) * \mathrm{y} / \mathrm{rIn}])$

$\mathrm{uz}=\mathrm{uz}$; ur $=\mathrm{ur} ; \mathrm{p}=\mathrm{p}$;

upz $=u p z ;$ upr $=$ upr; pp $=p p$;

$\mathrm{ThF}=\operatorname{adaptmesh}(\mathrm{ThF},[\mathrm{dx}(\mathrm{uz}), \mathrm{dy}(\mathrm{ur})]$,

hmin $=$ elementMinSize,

hmax $=$ elementMaxSize);

$\mathrm{t}-=$ coef;

\} else \{

$\mathrm{c} /=2.0$;

\}

\}

To implement the algorithm, the following FreeFem++ commands were used: checking the possibility of the appearance of negative volumes checkmovemesh, transforming the mesh movemesh and adapting the mesh adaptmesh (it is necessary for the sizes of the finite elements to lie within the specified range and the mesh density was proportional to the fluid velocity gradient). Assignment of the form $u z=u z$ is necessary to recalculate the value of the FEM variable on the updated mesh.

(a)

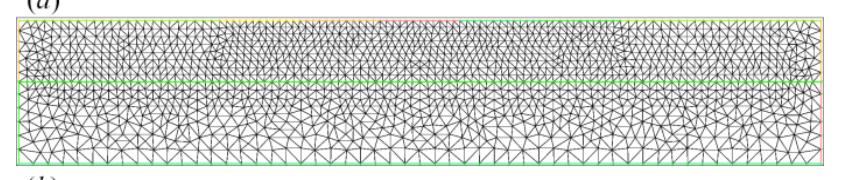

(b)

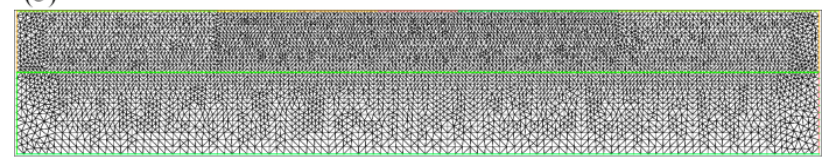

Fig. 3. Pipe (upper) and channel (lower) meshes $M\left(12,{ }^{*}, 5\right)(a)$ and $M\left(24,{ }^{*}, 5\right)(b)$.

3.3. The error analysis and the model applicability limits In a symmetric problem with one PEA, the average liquid flow rate over the period is equal to zero. To determine the systematic error (a nonzero average fluid flow rate), calculations were performed for various parameters of the mesh for a time interval corresponding to the characteristic relaxation time of the velocity [25] (equal to $1 \mathrm{~s}$ in the considered geometry):

$\tau_{u}=\rho R_{1}^{2} / \mu$.

For numerical simulation, the average flow rate is introduced as follows

$Q_{a}=\left(V^{n+1}-V^{n}\right) \tau$,

where $V^{n+1}$ and $V^{n}$ are the total volume of liquid passed through the outlet in the current and previous periods of oscillation, respectively.

To check the numerical scheme of the equations of hydrodynamics, a numerical simulation of the Poiseuille flow in a pipe with a circular cross section was carried out and a comparison with the analytical formula [32] was performed

$Q_{p}=\pi \Delta p R_{1}^{4} /(8 \mu L)$

Additionally, the analytical dependence of the liquid flow rate $Q_{a}$ on time is compared with a numerical simulation. The amplitude of the velocity versus time in the first approximation is [38]

$u(0, t)=\Delta p R_{1}^{2} g(t) /(4 \mu L)$,

where the function of time $g(t)$ is determined by the following formula:

$g(t)=1-\frac{(1-a) \exp \left(-\lambda_{1}^{2} t / \tau_{u}\right)}{8 \lambda_{1}^{3} J_{1}\left(\lambda_{1}\right)}$

$\lambda_{1} \approx 2.4$ is the first root of the Bessel function of the zero kind $J_{0}\left(\lambda_{1}\right)=0 ; J_{1}\left(\lambda_{1}\right) \approx 0.52$ is the Bessel function of the first kind; $a=0.113$ is an fitting parameter introduced to compensate the discarded expansion terms selected from the physical meaning: there is no flow at the initial time $u(0,0)=0\left(g(0)=0\right.$, then $\left.Q_{a}(0)=0\right)$, and there is reachment of constant flow rate $u(0, t)=$ const over the time interval $t>\tau_{u}\left(g(t) \rightarrow 1\right.$, then $\left.Q_{a}(t) \rightarrow Q_{p}\right)$. The liquid flow rate is related to the amplitude of the velocity $u(0, t)$ by the ratio $Q_{p}(t)=(\pi / 2) R_{1}^{2} u(0, t)$ :

$Q_{a}(t) \approx Q_{a} g(t)$

Fig. 4 shows the dependences of the average fluid flow rate $Q_{p}$ calculated by formula (13), the fluid flow rate calculated by formula (14), and the numerically obtained dependences $Q_{a}(t)$ for a pressure difference $\Delta p=0.5 \mathrm{~Pa}$ in the absence of oscillations of the PEA and when it oscillating with a frequency $f=1 \mathrm{kHz}$. It can be seen that during the characteristic velocity relaxation time $\tau_{u}$ calculated by formula (11) the values of $Q_{a}$ approach to the analytical value of $Q_{p}$; the analytical dependence $Q_{a}(t)$ and the numerical dependences $Q_{a}(t)$ with and without oscillations practically coincide.

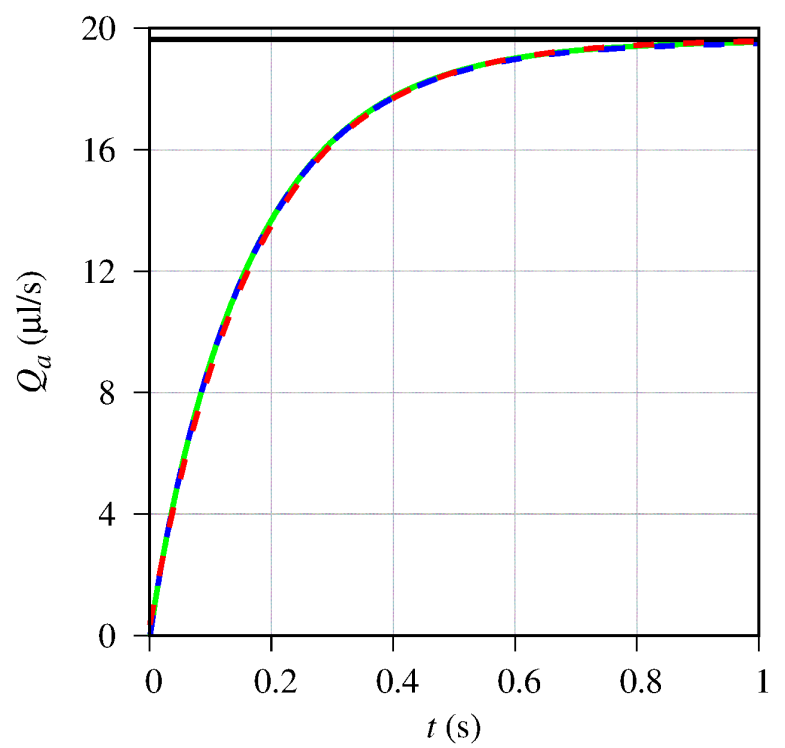

Fig. 4. Dependence of the average fluid flow rate $Q_{a}$ for a Poiseuille flow vs. time $t$ for analytical formula (13) (black line), formula (14) (red line), numerical simulation in the absence of oscillation of the PEA 
(green line) and when PEA oscillates (blue line) with frequency $f=1 \mathrm{kHz} ; \Delta p=0.5 \mathrm{~Pa}$.

Let's determine the sources of errors and their magnitude. The systematic error is equal to the steady-state average fluid flow rate $\varepsilon_{a i}=Q_{a}$, where $i=N$ for NBC and $i=D$ for $\mathrm{DBC}$. The relative error for the fluid flow rate is calculated by the formula

$\varepsilon_{Q}=\left|1+Q_{i} / Q_{o}\right|$

where $Q_{i}$ and $Q_{o}$ are the flow rates at the inlet and outlet, respectively. Note that the fluid flow rate is positive for the fluid flow in the positive direction of $O z$ and negative for the flow in the opposite direction, for example, during the compression of the tube $Q_{i}<0$ and $Q_{o}>0$.

Since the tube and the fluid in it are incompressible, the change of the channel volume must be equal to the volume of the fluid flowing through the ends, when the tube is deformed. The relative error in the volume change is described by the formula

$\varepsilon_{V}=\frac{\left|\left(Q^{i}+Q^{i+1}\right) \tau-\left(V_{e}^{i}-V_{e}^{i+1}\right)\right|}{V_{e}^{0}}$,

where the superscripts $i$ and $i+1$ correspond to the previous and current time steps; $V_{e}^{i}$ and $V_{e}^{0}$ are the volumes in the deformed and non-deformed tube, calculated by the formulas

$V_{e}^{i}=\int 2 \pi r \mathrm{~d} r \mathrm{~d} z, V_{e}^{0}=\pi\left(R_{2}^{2}-R_{12}^{2}\right) L$.

Here, the domain of integration $\Omega$ is bounded along the $O z$ axis by the interval $[0, L]$, and along the $O r$ axis places between the curves $R_{1}+s_{r}\left(z, R_{1}\right)$ and $R_{2}+s_{r}\left(z, R_{2}\right)$ with the radial component of deformation $s_{r}<0$ (the direction of the deformation and $\mathrm{Or}$ axis are opposite). In the simulations, the relative error $\varepsilon_{V}$ do not exceed $2.4 \cdot 10^{-12 \%}$ for DBC and $3.5 \cdot 10^{-12} \%$ for NBC.

Note that the time to reach a constant non-zero fluid flow rate and the time to establish a Poiseuille-type flow are close to the characteristic time $\tau_{u}$, which suggests the possibility of reducing the systematic error by specifying a constant pressure difference as follow

$\Delta p_{i}^{a}=-8 \mu \varepsilon_{i}^{a} L /\left(\pi R_{1}^{4}\right)$.

As a check, simulations were carried out with the parameters $M\left(12,20, N_{p}\right)$ for $N_{p}=[1,3,5]$ PEAs with NBC and $\mathrm{DBC}$ and frequency $f=1 \mathrm{kHz}$. The systematic error ranges from 2.2 to $72 \mathrm{nl} / \mathrm{s}$ depending on the parameters (see Table 1). To suppress this error, one need to set the pressure difference $\Delta p$ according to formula (14). Recalculation with a constant pressure difference significantly reduces the systematic error $\varepsilon_{a i}$ to hundredths or tenths of $\mathrm{nl} / \mathrm{s}$ (they are placed in the lines marked with $M^{*}$ in Table 1), which is 40 250 times less than the error of the initial simulation Note that it is difficult to further reduce the error, since, according to (12), the volume change per time step is of the order of $\Delta V_{m}=V^{n+1}-V^{n}=4 \cdot 10^{-17} \mathrm{~m}^{3}$, which is in the range of round-off errors. This value of the volume change $\Delta V_{m}$ determines the limits of the model applicability. For example, with decreasing frequency, if the volume change is of the order of $\Delta V_{m}$, then the simulation results will be incorrect, and if $\Delta V=k \Delta V_{m}$, then rounding errors in $Q_{a}$ will be order of $1 / k$. Simulations show that at a frequency of oscillations of PEA $f \leq 500 \mathrm{~Hz}$, the systematic errors will be $\varepsilon_{a i} \geq 10 \%$.

Table 1 show that increasing the time steps proportionally increases the calculation time, but slightly decreases the error. The increase in the number of FEMmesh elements proportionally increases the calculation time and reduces the calculation error. The CPU time was determined by a profiler written in the FreeFem++ language and distributed as follows: solving the hydrodynamic equations is $76 \%$, solving the elastic equations is $12.7 \%$, remeshing is $8.7 \%$. The remaining time $(2.6 \%)$ is spent on $\mathrm{I} / \mathrm{O}$ operations (writing 7 data files with a total size of $25 \mathrm{MB}$ and streaming $\log$-information to the console). The calculations were carried out on a 4-core Intel $i 7-3770 \mathrm{~K}$ processor in the Ubuntu 14.04 OS in the FreeFem++ 3.26 software.

\subsection{Micropump model}

As shown in Section 2.2, with symmetric compression of PEAs in the absence of a pressure difference, the average fluid flow is zero. In the micropump model, the flow was created using an asymmetric scheme of periodic compression of $N_{p}$ PEAs: first, all PEAs are simultaneously compressed during the time $T_{f} / 2$; then, a successive stretching of the PEAs one by one is performed starting from the leftmost one at each subsequent interval $T_{f} / 2$. Thus, the total time of the compression-expansion cycle of the system of PEAs is $T_{c}=T_{f}$ for $N_{p}=1, T_{c}=2 T_{f}$ for $N_{p}=3$, and $T_{c}=3 T_{f}$ for $N_{p}=5$; then the cycle was repeated. Fig. 5 shows the deformation steps for a system of three PEAs.

Fig. 6 shows the profiles of the outer boundary of the tube deformed by PEAs at the end of each half-period of operation of the system of five PEAs for NBC and DBC. $\mathrm{NBC}$ results in smoother tube deformation, while DBC results in stepwise deformation. This is explained by the fact that for the stability of the numerical scheme, the outer boundary of the tube $\Gamma_{6}$, which is not in contact with the PEAs, was also fixed in order to avoid conflict with the condition of rigid fixation of the tube at the ends. DBC provides exact value of maximum compression $s_{m}=\max \left(\left|s_{r}\right|\right)$ for each PEA. When using $N B C$, the exact value $s_{m}$ is ensured with the simultaneous compression of two or more PEAs. In the last period, when the penultimate PEA expands, $\left(\sim 0.1 s_{m}\right)$ stretching of the last PEA occurs. This is explained as follows. When a nonzero pressure is set on adjacent PEAs, deformation is provided by both PEAs in the region between the PEAs because the pressure jump is less than between the PEA and the free surface of the tube. At the same pressure, one PEA should deform the area to the left and right of itself, which leads to less compression of the tube in the middle of the contact area.

Fig. 7 shows the influence of the boundary conditions and the number of PEAs in the system on the flow created by the micropump. The amplitude of the fluid flow rate (Fig. 7(a)) with symmetrical compression of the PEAs system is proportional to the number of PEAs $N_{p}$ since the volume change during deformation of the tube is proportional to $N_{p}$, and the fluid is symmetrically pushed out of the tube. The amplitude of the liquid flow rate decreases significantly (by 3-6 times depending on the number of the half-period) with subsequent successive expands, and the liquid is asymmetrically drawn into the tube. 

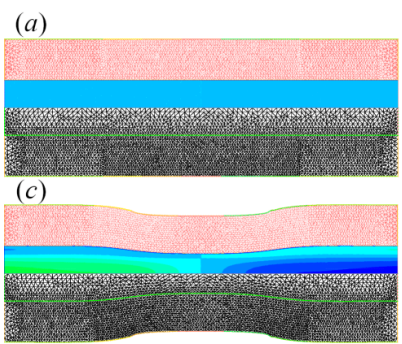

(e) (b)

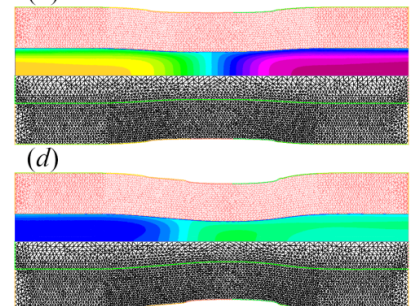

$(f)$
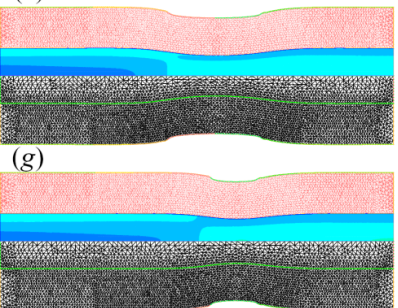

$u_{z} \quad-4$

$-2$

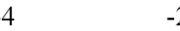

0

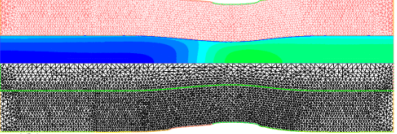

$(h)$

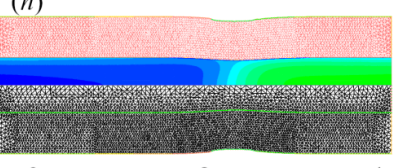

2
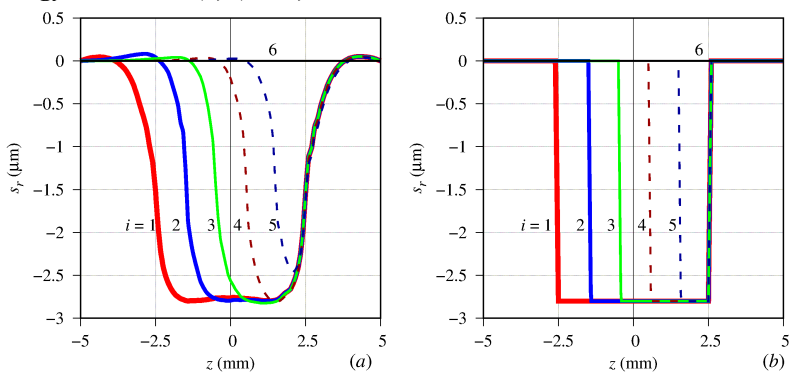

Fig. 6. Profiles of the outer surface of the tube for the NBC $(a)$ and DBC $(b)$ at the end of each half-period of PEAs oscillations $t=i T_{f} / 2$ in the case of $N_{p}=5$.

The total volume of fluid passing through the tube outlet (Fig. $7(b)$ ) varies over a period within $17 \%$ of the mean. With DBC compared to NBC, the total volume of fluid is more than twice larger due to the greater volume change during tube deformation. An increase in the number of PEAs in the system proportionally increases the volume of pumped fluid. Fig. 7(c) shows the time dependence of the average fluid flow rate $Q_{a}$. It is seen that in a time of the order of $\tau_{u} \approx 1 \mathrm{~s}$, the value of $Q_{a}$ reaches a stationary operating mode (filled area).
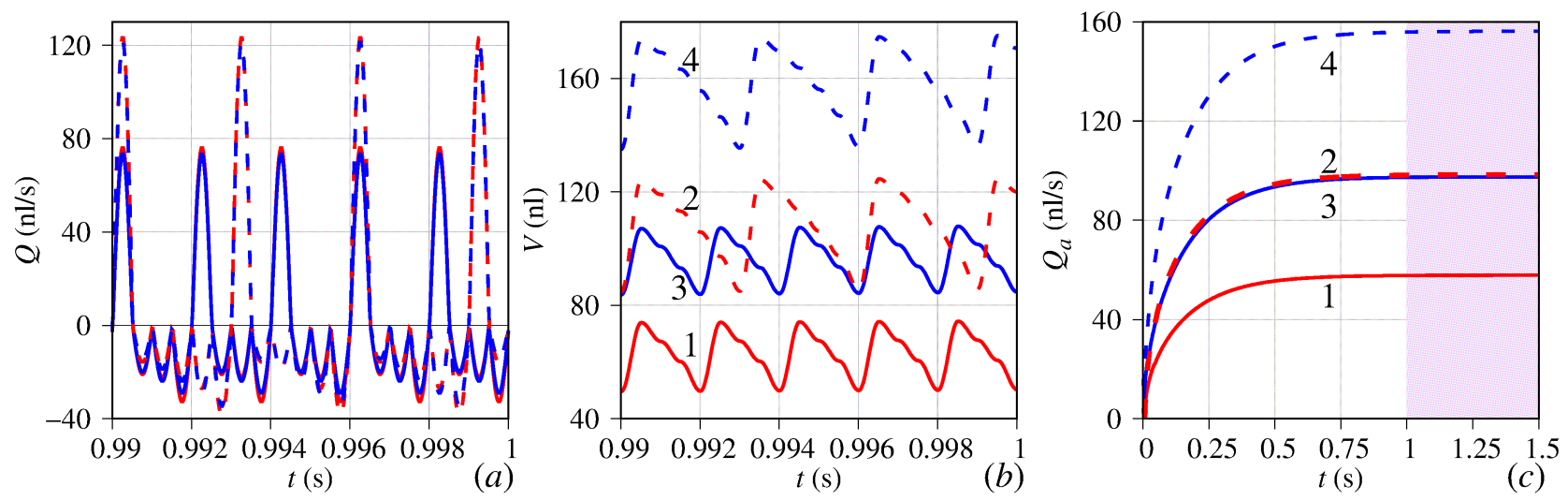

Fig. 7. Comparison of the $\operatorname{NBC}(1,2)$ and $\operatorname{DBC}(3,4)$ for $N_{p}=3(1,3)$ and $N_{p}=5(2,4)$. Depending on the time at the outlet $\Gamma_{2}$ : flow rate $Q(a)$, total volume of fluid $V(b)$; average fluid flow rate over the period $Q_{a}(c)$ for $f=1 \mathrm{kHz}$.

Fig. 8(a) shows the dependence of the steady-state fluid flow rate $Q_{a}$ on the oscillation frequency $f$. With an increase in the vibration frequency, the fluid flow rate increases nonlinearly. The vibration frequency dependence of the flow rate ratio for $N_{p}=5$ and $N_{p}=3$ PEAs defined as $N_{53}=Q_{a}\left(N_{p}=5\right) / Q_{a}\left(N_{p}=3\right)$ is shown in Fig. $8(b)$. With increasing frequency, this ratio slightly increases except for the region of high systematic errors $f \leq 500 \mathrm{~Hz}$.

Fig. 9(a) shows the dependence of the steady-state fluid flow rate $Q_{a}$ on the radius of the microchannel $R_{1}$. A decrease in $R_{1}$ leads to a decrease in the change in the microchannel volume during the oscillation period and, consequently, to a decrease in the value of $Q_{a}$.

The maximum radial compression PEA $s_{m}$ is selected for real models. When using technical solutions that enhance deformation, this parameter may increase. Fig. $9(b)$ shows the dependence of the average steady fluid flow rate $Q_{a}$ on the amplitude of radial compression. It can be seen that an increase of $s_{m}$ leads to a significant nonlinear increase in $Q_{a}$, which is explained by the fact that compressed PEAs create a higher hydraulic resistance.
Note that in modeling the PEAs system, the systematic error $\varepsilon_{a}$ is determined as the difference between the experimental $Q_{e}$ and numerical $Q_{n}$ values of the steady-state average fluid flow rate, i.e. $\varepsilon_{a}=Q_{e}-Q_{n}$. The systematic error can be compensated by an additional pressure difference $\Delta p$ determined by the approximate formula (16).

\subsection{Interaction of a micro pump with a micro-gripper}

The geometrical dimensions of the proposed micropump and the parameters of the operating mode make it possible to use the micropump for supplying coolant to the micro-gripper liquid cooling system. Let's estimate the heat distribution by the contact of the tube with the heated area near the outlet. The tube geometry expands by $\Delta L=2 \mathrm{~mm}$ at both ends. Since the length of the tube has changed, the new length denotes by $L_{1}=L+2 \Delta L$. TS, having a temperature $\Delta T_{0}=5 \mathrm{~K}$ above the ambient temperature $\left(T_{0}=293.15 \mathrm{~K}\right)$ and a length of $1 \mathrm{~mm}$, is placed at a distance of $1 \mathrm{~mm}$ from the tube outlet (see Fig. 10). Additional physical parameters of water are isobaric specific heat $c_{p}=4.183 \mathrm{~kJ} /(\mathrm{kg} \cdot \mathrm{K})$ and thermal conductivity $\lambda=0.599 \mathrm{Wt} /(\mathrm{m} \cdot \mathrm{K})$.

At the initial moment of time, the temperature of the fluid is the same and it is equal to $T_{0}$. A constant ambient 
Ildar Sh. Nasibullayev, Elvira Sh. Nasibullaeva and Oleg V. Darintsev/

Journal of Engineering Science and Technology Review 14 (2) (2021) 152 - 164

temperature $T_{0}$ is set at the inlet $\Gamma_{0}$; a constant temperature $T_{0}+\Delta T_{0}$ is set at the contact area of the tube with the TS $\Gamma_{7}$; no-temperature gradient condition is set at the outlet $\Gamma_{8}$ (boundaries are denoted on Fig. 1):

$$
\Gamma_{0}: T=T_{0} ; \quad \Gamma_{7}: T=T_{0}+\Delta T_{0} ; \quad \Gamma_{8}: \frac{\mathrm{d} T}{\mathrm{~d} z}=0
$$
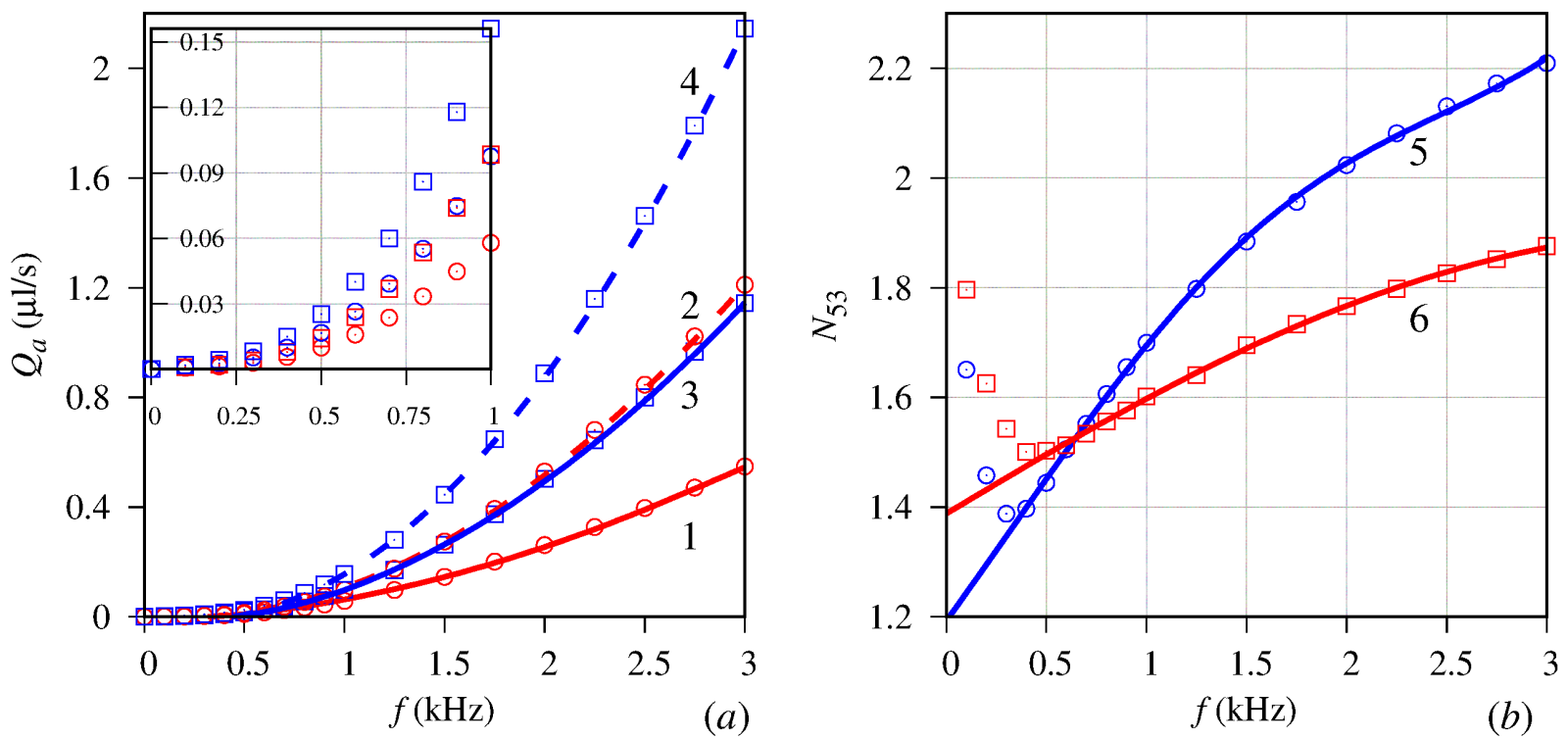

Fig. 8. The average fluid flow rate $Q_{a}(a)$ and $N_{53}(b)$ versus the oscillation frequency $f$ for the $\operatorname{NBC}(1,2,5)$ and $\operatorname{DBC}(2,4,6)$ for $N_{p}=3(1,3)$ and $N_{p}=5(2,4)$. Symbols are numerical results; lines are approximation (17).
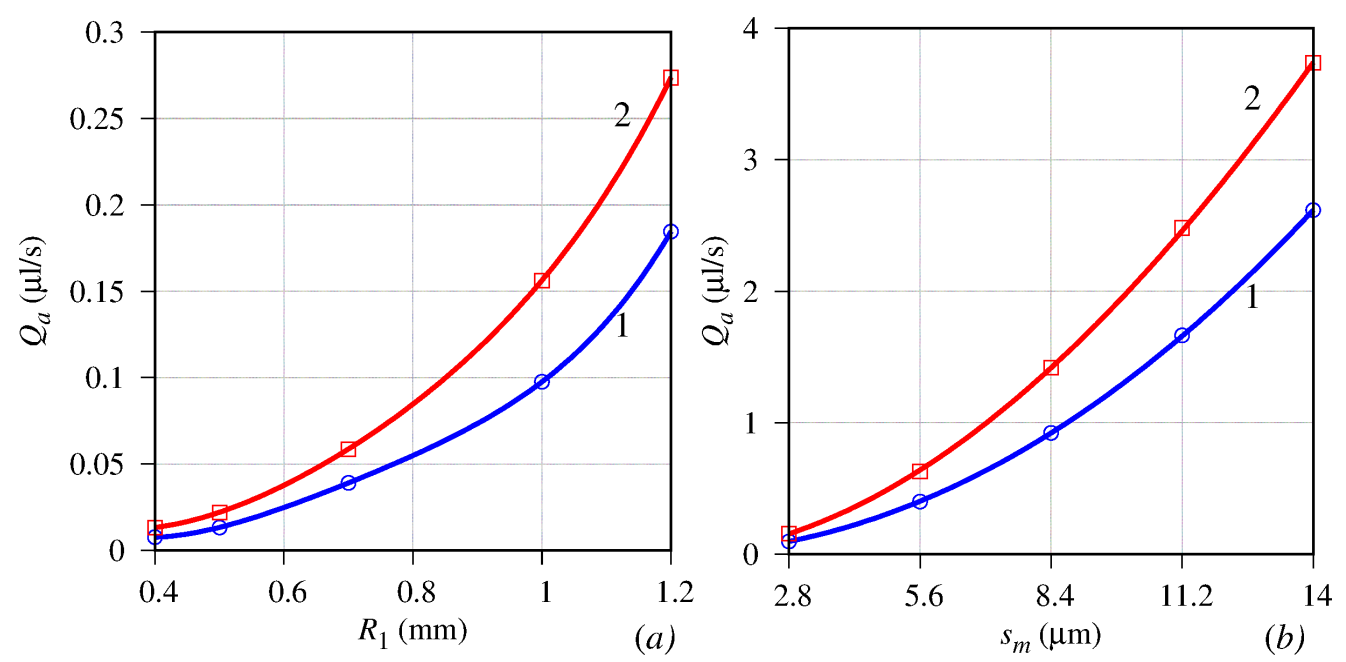

Fig. 9. The average fluid flow rate $Q_{a}$ versus the radius $R_{1}(a)$ and the strain amplitude $s_{m}(b)$ for the NBC $(1)$ and the DBC $(2)$ for $N_{p}=5 ; f=1 \mathrm{kHz}$. Symbols are numerical results; lines are approximation (17).

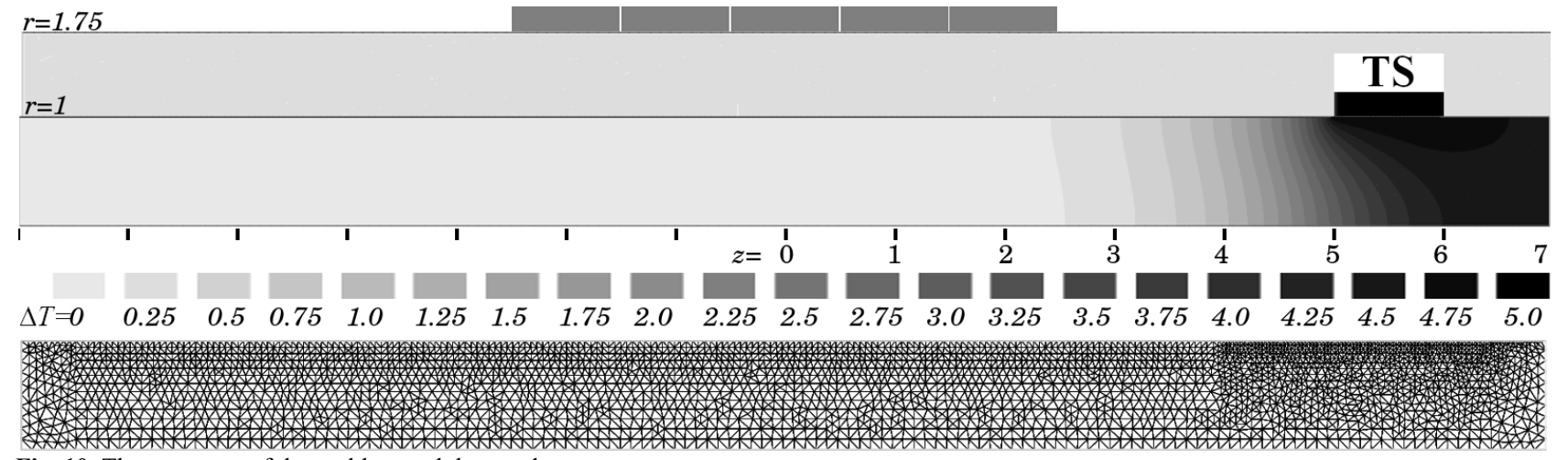

Fig. 10. The geometry of the problem and the steady-state temperature distribution $\Delta T$ for $Q_{a}=0.5 \mu \mathrm{l} / \mathrm{s}$ (up); the channel computational mesh (down) at time $t=120 \mathrm{~s}$. 

$L_{1}$.

The flow is induced by the pressure difference $\Delta p$ set at

Let's analyze the time dependence of the temperature distribution under the influence of the fluid flow. The Péclet number $\mathrm{Pe}=c_{p} \rho u R_{1} / \lambda$ allows one to determine the predominant nature of heat transfer (dynamic $\mathrm{Pe}>1$ or diffusion $\mathrm{Pe}<1$ ) [39]. Substituting the expression for the velocity amplitude $u=\Delta p R_{1}^{2} /(4 \mu L)$ with the pressure difference $\Delta p$ obtained from the expression for the average fluid flow rate $Q_{a}=\pi R_{1}^{4} \Delta p /(8 \mu L)$, the condition for dynamic heat transfer $\mathrm{Pe}=2 c_{p} \rho Q_{a} /\left(\pi \lambda R_{1}\right)>1$ is obtained, i.e. $Q_{a}>225 \mathrm{nl} / \mathrm{s}$.

Three series of simulations were performed:

1) full numerical simulation with a frequency $f=2 \mathrm{kHz}$ with DBC on the computational scheme $M(12,20,5)$;

2) the fluid at rest and heat from TS spreads only due to thermal conductivity (computational scheme $M(24,500)$ );

3) numerical simulation of the fluid flow induced by a constant pressure difference $\Delta p$, which corresponds to the average fluid flow rate $Q_{a}$, i.e. with the replacement of the micropump with the computing stand element (CSE) (computational scheme M(24,500)).

The latter option makes it possible to significantly reduce the calculation time due to the fact that in the absence of tube deformations it is not necessary to solve the equations of linear elasticity, which is uses $\sim 18 \%$ of the total calculated time; the time step can be chosen equal to $\tau_{d} / N$ $\left(N=500\right.$ was used in the calculations) instead of $\tau_{d} /(6 f N)$ (in the simulations of the complete numerical problem the frequency $f=2000 \mathrm{~Hz}$ and sub-cycle time steps $N=20$ with 6 oscillation sub-cycles were used). Additional savings in the estimated time was achieved due to the fact that after a certain time $\left(1.5 \tau_{u}=1 \mathrm{~s}\right.$ was used in the simulations $)$ a stationary flow is established, and the subsequent solution of the hydrodynamic equations is not required $(\sim 70 \%$ of the total simulation time), only the heat conduction equation is solved for a given fluid velocity profile $(\sim 10 \%$ of the total simulation time). For comparison, the complete numerical simulation for the physical time interval of $5.9 \mathrm{~s}$ was $\sim 37 \mathrm{~h}$ of the CPU time, and the numerical simulation of the same time interval using CSE was $\sim 7$ min of the CPU time, i.e. the CPU time has decreased by about $10^{4}$ times.

At the initial moment, the fluid has an ambient temperature $T=T_{0}$. Heat begins to spread from TS into the microchannel with time. Fig. 11 shows the time dependence of the fluid temperature on the axis $r=0$ at points $z=3,4 \mathrm{~mm}$ for three types of simulations. In the absence of a flow, the temperature spreads symmetrically with respect to the TS and grows exponentially with time at the points under study. The symmetry is broken under non-zero flow because the heated fluid is displaced by the flow of colder fluid. The full numerical simulation for $Q_{a}=754 \mathrm{nl} / \mathrm{s}$ is in qualitative agreement with the simulation using CSE for $Q_{a}=500 \mathrm{nl} / \mathrm{s}$ with an accuracy of $10 \%$. The differences in values of $Q_{a}$ are explained by the fact that, in contrast to the full simulation, in the CSE-simulation the fluid flows only in one direction with a constant velocity, i.e. there is no additional thermal diffusion which appears then the flow direction is reversed. The Péclet number in this calculation is $\mathrm{Pe}=2.2$, i.e. the contribution of fluid flow to heat transfer is higher than thermal diffusion. This is clearly seen on Fig 10 from the stationary-state temperature field $\Delta T$ (process time $t=120 \mathrm{~s}$ ) because the transverse temperature profile $\Delta T$ bends in the positive direction near the tube axis, where the fluid velocity is maximum.

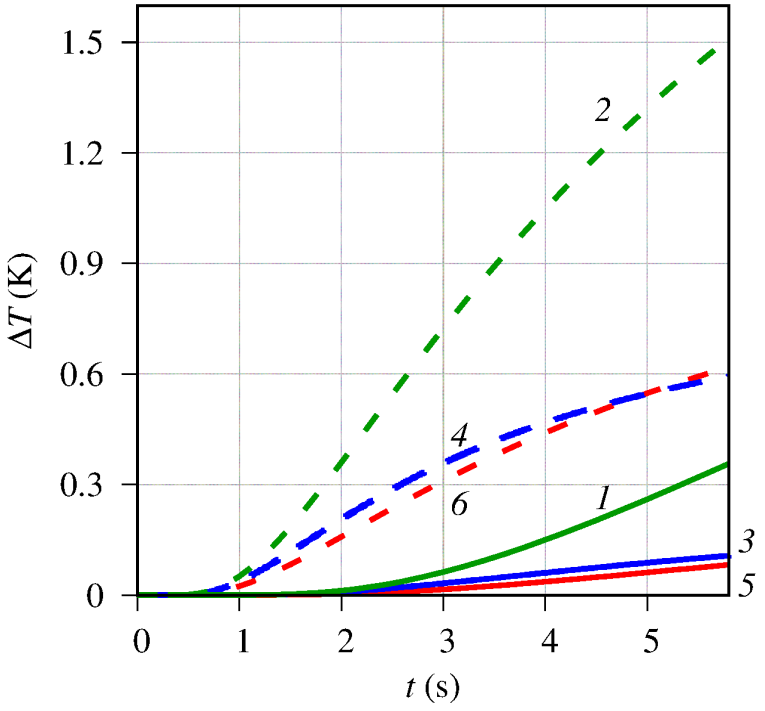

Fig. 11. Dependence $\Delta T$ at points $z=3 \mathrm{~mm}(1,3,5)$ and $z=4 \mathrm{~mm}(2,4,6)$ on the axis $r=0$ versus time $t$ for the no-flow case $(1,2)$, full numerical simulation $\left(N_{p}=5 ; f=2 \mathrm{kHz}\right.$; DBC; 3, 4) and simulation of the $\operatorname{CSE}\left(Q_{a}=0.5 \mu \mathrm{l} / \mathrm{s} ; 5,6\right)$.

Numerical simulation of the steady-state values of $\Delta T$ for the set of coordinates $z=2,3,4,5,6,7 \mathrm{~mm}$ on the axis $r=0$ versus $Q_{a}$ using CSE are shown on Fig. 12. It can be seen that at $\mathrm{Pe}<1$ the steady-state temperature in the region to the left of the TS $(z \leq 4 \mathrm{~mm})$ is inversely proportional to $Q_{a}$ due to diffusion type of the heat transfer (a linear temperature distribution is established from the inlet with temperature $T_{0}$ to the TS with temperature $T_{0}+\Delta T_{0}$ ). With an increase in the fluid flow rate (and the fluid velocity), the region of heat propagation is "compressed" close to the TS. Starting from the value of $\mathrm{Pe}=1 \quad\left(Q_{a}=225 \mathrm{nl} / \mathrm{s}\right)$ the dependence becomes nonlinear. Namely, the steady-state temperature in the region of the most PEA equal to $1.05 \mathrm{~K}$ $\left(21 \% \Delta T_{0}\right)$; at $\mathrm{Pe}=2$ in the same point decreases to the value of $0.27 \mathrm{~K}\left(5.4 \% \Delta T_{0}\right)$; at $\mathrm{Pe}=3$ the temperature is equal to $T(z=2)=0.067 \Delta T_{0} \quad\left(1.3 \% \Delta T_{0}\right) ; \quad$ and at $\mathrm{Pe}=4$ the temperature change is neglectable $T(z=2)=0.017 \Delta T_{0}$ $\left(0.3 \% \Delta T_{0}\right)$. In the region to the right of the TS $z \geq 5 \mathrm{~mm}$ the fluid temperature increases both by diffusion heat transfer and the fluid flow. It can be seen on Fig. 12, where for $\mathrm{Pe}<1$ the temperature change $\Delta T$ with increasing $Q_{a}$ is nonlinear (both diffusion heat transfer and fluid flow), and for $\mathrm{Pe}>1$ it is close to linear (proportional to the fluid flow rate).

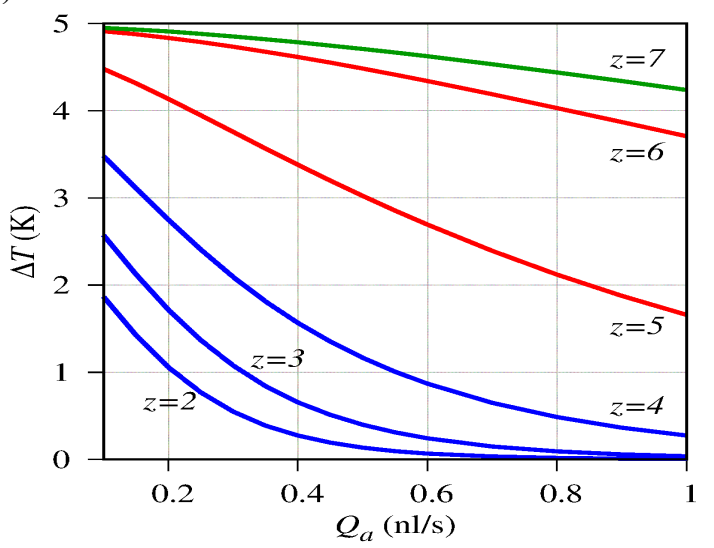

Fig. 12. Dependence of steady-state heating $\Delta T$ on the $r=0$ axis at points to the left of the TS $(z=2,3,4 \mathrm{~mm}$; blue lines), under the TS 
Ildar Sh. Nasibullayev, Elvira Sh. Nasibullaeva and Oleg V. Darintsev/

Journal of Engineering Science and Technology Review 14 (2) (2021) 152 - 164

$(z=5,6 \mathrm{~mm}$; red lines) and at the channel outlet $(z=7 \mathrm{~mm}$; green line $)$ versus the average fluid flow rate $Q_{a}$.

Thus, the proposed micropump design can be used to cool the micro-gripper when operates with $N_{p}=5$ PEAs at a frequency of $f>2.5$ for $\mathrm{NBC}$ or $f>2 \mathrm{kHz}$ for $\mathrm{DBC}$.

\subsection{Computational stand element of the micropump}

Numerical simulation is time consuming (see Table 1) and cannot provide real-time information to control the pump. Therefore, on the basis of numerical simulation, a simple analytical approximation was derived for the fluid flow rate as a function of the boundary conditions, frequency $f$, the number of PEAs $N_{p}$, the inner radius $R_{1}$ and the amplitude of radial compression $s_{r}^{m}$ :

$Q_{a}=g(f) g\left(N_{i j}\right) g\left(R_{1}\right) g\left(s_{m}\right)$

physical meaning of the dependence of $Q_{a}$ on the arguments. Since with an increase in the frequency $f$ and the amplitude of radial compression $s_{m}$, the fluid flow rate becomes linear, the functions $g(f)$ and $g\left(s_{m}\right)$ are determined by the interpolations as follow

$$
\begin{aligned}
& g(f)=a_{f} f\left(1-\frac{f}{1+b_{f} f}\right), \\
& g\left(s_{m}\right)=a_{s} s_{m}\left(1+\frac{10^{3} s_{m}}{1+b_{s} s_{m}}\right)
\end{aligned}
$$

with $a_{f}=7.78 \cdot 10^{-12}, \quad b_{f}=2.05 \cdot 10^{-5}, \quad a_{s}=-0.529, \quad b_{s}=-$ $2.52 \cdot 10^{-6}$ for $\mathrm{NBC}$ and $a_{f}=9.16 \cdot 10^{-11}, b_{f}=3.08 \cdot 10^{-6}$, $a_{s}=0.107, b_{s}=1.4 \cdot 10^{-5}$ for $\mathrm{DBC}$.

where the functions $g$ were selected according to the

Table 1. Errors and CPU time of the simulation versus simulation parameters and boundary conditions: $\varepsilon_{a D}$ and $\varepsilon_{a N}$ are systematic errors $Q_{a}$ for the $\mathrm{DBC}$ and the $\mathrm{NBC}$, respectively; $\varepsilon_{Q D}$ and $\varepsilon_{Q N}$ are relative errors of the inlet and outlet flow rate for the DBC and the NBC calculated by (15); $N_{e}$ and $N_{l}$ are numbers of the mesh elements for pipe and channel, respectively; $T_{e}$, $T_{r}, T_{l}, T_{t}$ are CPU times used for Lame equations, remeshing, Navier-Stokes equations and total time, respectively; $M^{*}$ is

\begin{tabular}{|c|c|c|c|c|c|c|c|c|c|c|}
\hline$M\left(n, m, N_{p}\right)$ & $\varepsilon_{a N}, \mathbf{n l} / \mathbf{s}$ & $\varepsilon_{Q N}, \%$ & $\varepsilon_{a D}, \mathrm{nl} / \mathrm{s}$ & $\varepsilon_{Q D}, \%$ & $N_{e}$ & $N_{l}$ & $T_{e}, \mathbf{s}$ & $T_{r}, \mathbf{s}$ & $T_{l}, \mathbf{s}$ & $T_{t}, \mathbf{s}$ \\
\hline$M(12,20,1)$ & 3.41 & 0.220 & -19.9 & 1.76 & & & & & & \\
\hline$M^{*}(12,20,1)$ & 0.0236 & & 0.0786 & & & & & & & \\
\hline$M(12,20,3)$ & -2.19 & 0.102 & -7.89 & 0.0521 & & & 104 & hao & $h_{2}$ & 204 \\
\hline$M^{*}(12,20,3)$ & -0.0276 & & -0.0485 & & & & 484 & 328 & 2363 & 3264 \\
\hline$M(12,20,5)$ & -31.9 & 0.099 & -72.1 & 0.0246 & 1850 & 1316 & & & & \\
\hline$M^{*}(12,20,5)$ & -0.791 & & -0.825 & & & & & & & \\
\hline$M(12,40,1)$ & 3.60 & 0.0774 & -19.7 & 1.76 & & & & & & \\
\hline$M(12,40,3)$ & -1.06 & 0.103 & -6.45 & 0.0535 & & & 959 & 652 & 4682 & 6516 \\
\hline$M(12,40,5)$ & -28.6 & 0.100 & -68.6 & 0.0259 & & & & & & \\
\hline$M(18,20,1)$ & -4.23 & 0.0748 & -16.3 & 1.19 & & & & & & \\
\hline$M(18,20,3)$ & 4.25 & 0.121 & -23.0 & 0.0075 & 3974 & 2635 & 1076 & 681 & 5160 & 7098 \\
\hline$M(18,20,5)$ & -5.53 & 0.0159 & -23.5 & 0.0252 & & & & & & \\
\hline$M(24,20,1)$ & -0.693 & 0.0139 & -11.4 & 0.899 & & & & & & \\
\hline$M(24,20,3)$ & -0.775 & 0.0179 & 4.80 & 0.0105 & 7276 & 5218 & 1945 & 1323 & 11660 & 15261 \\
\hline$M(24,20,5)$ & -13.8 & 0.0249 & -9.98 & 0.0154 & & & & & & \\
\hline
\end{tabular}
compensation of the systematical error by formula (16). Parameters: $f=1 \mathrm{kHz} ; t=1 \mathrm{~s}$

The function $g\left(N_{i j}\right)=Q_{a}\left(N_{p}=i\right) / Q_{a}\left(N_{p}=j\right) \quad$ was approximated by a third order polynomial using the least squares method (LSM) [40], and the function $g\left(R_{1}\right)$ was approximates by a fourth order polynomial using the LSM due to the analytical formula for the Poiseuille flow in a pipe with circular cross-section has $R_{1}^{4}$ term [20]:

$$
\begin{aligned}
& g\left(N_{i j}\right)=\sum_{k=0}^{3} a_{k}\left(\frac{Q_{a}\left(N_{p}=i\right)}{Q_{a}\left(N_{p}=j\right)}\right)^{k}, \\
& g\left(R_{1}\right)=\sum_{k=0}^{4} b_{k} R_{1}^{k}
\end{aligned}
$$

with $a_{0}=0.839, \quad a_{1}=-3.58 \cdot 10^{-4}, \quad a_{2}=1.25 \cdot 10^{-7}, \quad a_{3}=-$ $1.64 \cdot 10^{-11}, b_{0}=1.79, b_{1}=-1.14 \cdot 10^{-2}, b_{2}=2.63 \cdot 10^{-5}, b_{3}=-$
$2.43 \cdot 10^{-8}, b_{4}=8.63 \cdot 10^{-12}$ for NBC and $a_{0}=0.72, a_{1}=-$ $1.14 \cdot 10^{-4}, a_{2}=2.06 \cdot 10^{-8}, a_{3}=-1.1 \cdot 10^{-12}, b_{0}=0.77, b_{1}=-$ $4.7 \cdot 10^{-3}, b_{2}=1.04 \cdot 10^{-5}, b_{3}=-8.72 \cdot 10^{-9}, b_{4}=3.21 \cdot 10^{-12}$ for DBC.

The approximations by the analytical formula (17) are shown by lines on the Fig. 8 and Fig. 9. It can be seen that the formula (17) computes the average steady-state fluid flow rate $Q_{a}$ with high accuracy. The time of reaching the operating mode $\tau_{u}$ (period-average steady-state) and the dynamics of the process are described by formulas (11) and (14), respectively. The formula (17) can be used as a CSE of the micropump-microgripper system as well as used in the software module of the micropump real-time control system. 


\section{Conclusion}

An axisymmetric mathematical and computer model of a piezoelectric micropump, consisting of an elastic tube and a system of PEAs, has been developed. The sources of error are analyzed (a method for reducing the systematic error by two orders of magnitude is proposed) and the region of the model applicability is determined $(f=0.5, \ldots, 100 \mathrm{kHz})$. The parameters of the micropump operating mode were obtained (time $\approx 1 \mathrm{c}$ and average fluid flow rate $Q_{a}$ up to $2 \mu \mathrm{l} / \mathrm{s}$ ). It is shown that $Q_{a}$ is proportional to the oscillation frequency of the PEAs and their number. $Q_{a}$ nonlinearly increases with an increase in the inner radius of the tube and the amplitude of the radial compression of the PEAs. DBCs provide double increase of the $Q_{a}$ over NBC. Average steady-state fluid flow rate $Q_{a}$ from $500 \mathrm{nl} / \mathrm{s}$ (for $f>2.5 \mathrm{kHz}$ at $\mathrm{NBC}$; for $f>2 \mathrm{kHz}$ at $\mathrm{DBC}$ ) prevents heat spreading from microgripper inside the micropump tube. Simulations have shown that the proposed model can be used to develop a compact micropump with a flexible control system of fluid flow rate for cooling a microgripper. The analytical formula (17) for calculating $Q_{a}$ depending on the boundary conditions, frequency, the number of PEAs, the inner radius and the amplitude of radial compression is constructed. This formula can be used in a real-time device control system.

\section{Acknowledgements}

This research was supported by funds of state assignment N. 0246-2018-007.

This is an Open Access article distributed under the terms of the Creative Commons Attribution License.

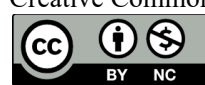

\section{References}

1. N. Convery and N. Gadegaard, "30 years of microfluidics". Micro and Nano Engineering, 2, $76 \quad$ (2019). DOI: 10.1016/j.mne.2019.01.003.

2. "Microfluidics Based Microsystems: Fundamentals and Applications" (eds. by S. Kakaç, B. Kosoy, D. Li and A. Pramuanjaroenkij), Springer (2010). DOI: 10.1007/978-90-4819029-4.

3. "Microsystems for Pharmatechnology. Manipulation of Fluids, Particles, Droplets, and Cells" (ed. by A. Dietzel), Springer (2016). DOI: 10.1007/978-3-319-26920-7.

4. "Cooling of Electronic Systems" (eds. by S. Kakaç, H. Yüncü and K. Hijikata), Springer, Netherlands (1994). DOI: 10.1007/978-94011-1090-7.

5. R.M. Cotta, D.C. Knupp and C.P. Naveira-Cotta, "Analytical Heat and Fluid Flow in Microchannels and Microsystems", Springer (2016). DOI: 10.1007/978-3-319-23312-3.

6. J. Berg and T. Dallas, "Peristaltic Pumps" in: Encyclopedia of Microfluidics and Nanofluidics (ed. by D. Li), New York, Springer (2015).

7. "MEMS. Applications" (ed. by M. Gad-el-Hak), CRC Press (2005).

8. J.J. Abbott, Z. Nagy, F. Beyeler and B.J. Nelson, "Robotics in the Small, Part I: Microbotics," in IEEE Robotics \& Automation Magazine, 14(2), 92 (2007). DOI: 10.1109/MRA.2007.380641.

9. Zh. Zhang, X. Wang, J. Liu, Ch. Dai and Yu Sun, "Robotic Micromanipulation: Fundamentals and Applications". Annual Review of Control, Robotics, and Autonomous Systems 2(1), 181 (2019)

10. O.V. Darintsev, A.B. Migranov, "Capillary micro-grip with feedback". RF Patent 2261795 RU (2005).

11. F. Afshari, "Experimental and numerical investigation on thermoelectric coolers for comparing air-to-water to air-to-air refrigerators". J. Therm. Anal. Calorim. (2020). DOI: 10.1007/s10973-020-09500-6.

12. O.V. Darintsev, "Specificity of work and design features of microgripping devices of robots". Proc. of Congress on Fundamental Problems of Theoretical and Applied Mechanics, 1, 43 (2019) (in Russian). DOI: 10.22226/2410-3535-2019-congress-v1.

13. A.B. Migranov, O.V. Darintsev and D.R. Bogdanov, "The specificity of the mechanics of the size reduced version of the capillary micro-capture". Proc. of Congress on Fundamental Problems of Theoretical and Applied Mechanics, 1, 581 (2019) (in Russian). DOI: 10.22226/2410-3535-2019-congress-v1.

14. O.V. Darintsev, D.R. Bogdanov and E.O. Darintseva, "Information support of an intelligent control system for a capillary microgripper". Proc. of Information Technologies for Intelligent Decision Making Support (ITIDS' 2014), 2, 242 (2014) (in Russian).

15. O.V. Darintsev, D.R. Bogdanov and E.O. Darintseva, "Multichannel systems for collecting and processing information for a semi-natural stand: architecture and processing algorithms". Proc. of the Mavlyutov Institute of Mechanics, 10, 44 (2014) (in Russian). DOI: 10.21662/uim2014.1.008.

16. D.J. Laser and J.G. Santiago, "A review of micropumps". Journal of Micromechanics and Microengineering, 14(6) (2004), R35. DOI: 10.1088/0960-1317/14/6/R01

17. "Fluid-structure Interaction: Modelling, Simulation, Optimization" (eds. by H.-J. Bungartz and M. Schäfer), Heidelberg, Springer (2006).

18. G. Hou, J. Wang and A. Layton, "Numerical Methods for FluidStructure Interaction - A Review". Communications in Computational Physics, 12(2), $337 \quad$ (2012). DOI: $10.4208 /$ cicp.291210.290411s.

19. G. Sandberg, P.-A. Wernberg and P. Davidsson, "Fundamentals of Fluid-Structure Interaction". In: G. Sandberg and R. Ohayon (eds) Computational Aspects of Structural Acoustics and Vibration. CISM International Centre for Mechanical Sciences, vol. 505. Springer, Vienna (2009). DOI: 10.1007/978-3-211-89651-8_2.

20. H. Bruus, "Theoretical microfluidics. Lecture notes third edition", Denmark, MIC Department of Micro and Nanotechnology Technical University of Denmark (2006).

21. Ch.-Yu Chiang, O. Pironneau, T. Sheu and M. Thiriet, "Numerical Study of a 3D Eulerian Monolithic Formulation for Incompressible Fluid-Structures Systems". Fluids, 2(2), $34 \quad$ (2017). DOI: 10.3390/fluids2020034.

22. F. Hecht, "New development in FreeFem++". J. Numer. Math., 20(3-4), 251 (2012). DOI: 10.1515/jnum-2012-0013.

23. J.O. Kim, J.G. Lee, "Dynamic characteristics of piezoelectric cylindrical transducers with radial polarization". Journal of Sound and Vibration, 300(1-2), $241 \quad$ (2007). DOI: $10.1016 /$ j.jsv.2006.08.021.

24. I.Sh. Nasibullayev and E.Sh. Nasibullaeva, "Fluid flow through the hydraulic resistance with a dynamically variable geometry". Proceedings of the Mavlyutov Institute of Mechanics,12(1), 59 (2017) (in Russian). DOI: 10.21662/uim2017.1.009.

25. I.Sh. Nasibullayev, E.Sh. Nasibullaeva and O.V. Darintsev, "Study of fluid flow through a channel deformed by piezoelement". Multiphase Systems, 13(3), 1 (2018) (in Russian). DOI: $10.21662 / \mathrm{mfs} 2018.3 .001$.

26. I.Sh. Nasibullayev, E.Sh. Nasibullaeva and O.V. Darintsev, "Simulation of fluid flow through a elastic microchannel deformed by a piezoelement in microgrip cooling systems". Mekhatronika, Avtomatizatsiya, Upravlenie, 20(12), 740 (2019) (in Russian). DOI: $10.17587 / \mathrm{mau} .20 .740-750$.

27. I.Sh. Nasibullayev and O.V. Darintsev, "Two-dimensional dynamic model of the interaction of a fluid and a piezoelectric bending actuator in a plane channel". Multiphase Systems, 14(4), 220 (2019) (in Russian). DOI: 10.21662/mfs2019.4.029.

28. I.Sh. Nasibullayev, O.V. Darintsev, E.Sh. Nasibullaeva and D.R. Bogdanov. "Piezoelectric Micropumps for Microrobotics: Operating Modes Simulating and Analysis of the Main Parameters of the Fluid Flow Generation". In: A. Ronzhin and V. Shishlakov (eds) Proceedings of 15th International Conference on Electromechanics and Robotics "Zavalishin's Readings". Smart Innovation, Systems and Technologies, Springer, Singapore, 187, 525 (2021). DOI: 10.1007/978-981-15-5580-0_43. 
Ildar Sh. Nasibullayev, Elvira Sh. Nasibullaeva and Oleg V. Darintsev/

\section{Journal of Engineering Science and Technology Review 14 (2) (2021) 152 - 164}

29. U.P. Adler, E.V. Markova and U.V. Granovskiy, "Design of experiment when selecting optimal conditions", Moscow, Nauka publ., 1976 (in Russian).

30. I.Sh. Nasibullayev, "The development of a computer model for the main element of the fuel metering unit". Computational Technologies, 21(2), 26 (2016) (in Russian).

31. C. Fütterer, N. Minc, V. Bormuth V, et al, "Injection and flow control system for microchannels". Lab Chip,. 4(4) 351 (2004). DOI:10.1039/b316729a.

32. L.D. Landau and E.M. Lifshitz, "Course of Theoretical Physics. Volume 6. Fluid Mechanics". Pergamon Press, 1987.

33. L.D. Landau and E.M. Lifshitz, "Course of Theoretical Physics. Volume 7. The theory of elasticity". Butterworth-Heinemann, 1986.

34. J. Xu, S. Lin, Y. Ma, and Y. Tang, "Analysis on Coupled Vibration of a Radially Polarized Piezoelectric Cylindrical Transducer". Sensors, 17(12), 2850 (2017). DOI: 10.3390/s17122850.
35. W. Wang, W. Shi, P. Thomas, and M. Yang, "Design and Analysis of Two Piezoelectric Cymbal Transducers with Metal Ring and Add Mass". Sensors, 19(1), 137 (2019). DOI: 10.3390/s19010137.

36. E. Murimi, J. Kihiu, G. Nyakoe, S. Mutuli, and J. Kimotho, "Determination of resonant frequency of a piezoelectric ring for generation of ultrasonic waves". Innovative Systems Design and Engineering, 2(4), 103 (2011).

37. P.J. Roache, "Computational Fluid Dynamics". Hermosa Publishers, 1976.

38. G.K. Batchelor, "An introduction to fluid dynamics". Cambridge University Press, 2000

39. S.V. Patankar, "Numerical heat transfer and fluid flow". Taylor and Francis, 1980.

40. "Handbook of Linear Algebra, 2nd Edition" (eds. by L. Hogben), CRC Press (2014). 